\title{
ENFRENTANDO AL COVID-19: SITUACIÓN DE LAS PERSONAS CON AUTISMO Y SUS FAMILIAS EN LATINOAMÉRICA
}

\section{Facing COVID-19: Situation of People with Autism and their Families in Latin America}

Daniel VALDEZ

FLACSO. Buenos Aires. Argentina

daniel.valdez@me.com

Cecilia Montiel Nava

University of Texas Rio Grande Valley. Edinburg. Texas

Cristina Silvestre Paula

Universidade Presbiteriana Mackenzie (UPM). São Paulo. Brasil

Alexia RatTazzi

PANAACEA. Buenos Aires. Argentina

Analía Rosoli

República Dominicana

Natalia BARRIOS

PANAACEA. Buenos Aires. Argentina

Sebastián Cukier

Argentina

Ricardo García

Universidad de Chile

Sandra MAnRique

CPAL. Perú

Georgina Pérez Liz

Drexel University. Filadelfia

Claudete VeIga de Lima

Universidade Presbiteriana Mackenzie (UPM). São Paulo. Brasil 
Cecilia Amigo

Universidad de la República. Montevideo. Uruguay

Virginia BEsIo

Universidad de la República. Montevideo. Uruguay

Gabriela GARRIDO

Universidad de la República. Montevideo. Uruguay

Recepción: 30 de octubre de 2020

Aceptación: 25 de enero de 2021

Resumen: Presentamos un estudio multicéntrico realizado a través de una encuesta en línea a 1826 familias de personas con TEA de Argentina, Brasil, Chile, México, Perú, República Dominicana, Uruguay y Venezuela. Nuestro objetivo es describir el impacto de la pandemia -y el aislamiento social- en la conducta, el estado de ánimo, el sueño y la alimentación de las personas con TEA. A tal fin hemos relevado características sociodemográficas, habitacionales y modalidad de confinamiento. Analizamos los efectos de la discontinuidad de los servicios educativos y terapéuticos y valoramos los alcances de las intervenciones a distancia. Algunas de las consecuencias del confinamiento -obligatorio para la mayoría- han sido el aumento de irritabilidad en las personas con TEA, el incremento de la conducta de deambular, mayores niveles de ansiedad, dificultades en alimentación, sueño y concentración. La mayor parte de las familias han notado retrocesos en sus hijos durante el encierro. Se destacan los efectos beneficiosos de salidas y paseos. Muchos tratamientos y clases se han suspendido. Se subrayan positivamente las intervenciones a distancia. La crisis actual debería ser una oportunidad para reorganizar dispositivos de educación y tratamiento, atendiendo a la necesidad de cambios, con una perspectiva más ecológica, inclusiva y amigable con el autismo.

Palabras clave: autismo; TEA; COVID-19.

Авstract: We present a multicenter study conducted through an online survey of 1826 families of people with ASD from Argentina, Brazil, Chile, Mexico, Peru, the Dominican Republic, Uruguay and Venezuela. Our objective is to describe the impact of the pandemic -and social isolation- on the behavior, mood, sleep and diet of people with ASD. To this end, we have surveyed sociodemographic and housing characteristics and the modality of confinement. We analyze the effects of discontinuity in educational and therapeutic services and assess the scope of remote interventions. Some of the consequences of confinement -obligatory for the majority- has been increased irritability in people with ASD, increased wandering behavior, higher levels of anxiety, and difficulties in eating, sleeping and concentrating. Most of the families have noticed setbacks in their children during the confinement. The beneficial effects of outings and walks are highlighted. Many treatments and classes have been discontinued. Remote 
interventions are positively highlighted. The current crisis should be an opportunity to reorganize education and treatment devices, attending to the need for changes, with a more ecological, inclusive and autism-friendly perspective.

KeYwords: autism; ASD; COVID-19.

\section{Introducción}

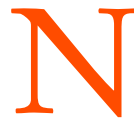

UESTRO MUNDO se encuentra en estado de emergencia, a causa de una pandemia producida por el COVID-19 que está poniendo a prueba la fortaleza del ser humano no solo a nivel sanitario, sino también en el plano emocional, social y económico. Es un gran desafío para todos avanzar en tiempos de incertidumbre. La incertidumbre nos hace más vulnerables frente a la ansiedad, al miedo, al cansancio, al encierro (Becker y Gregory, 2020). Para los niños, jóvenes y adultos con trastornos del espectro del autismo (TEA), afrontar esta situación puede resultar todavía más difícil. Es posible que surjan nuevas barreras frente a la exigencia de adaptaciones dinámicas a los cambios, a la necesidad de flexibilidad, a las posibilidades de comunicar y compartir las experiencias internas o las propias necesidades.

Ante lo inédito de esta situación urge crear dispositivos de apoyo para las personas con TEA y sus familias. Mantener rutinas, promover los vínculos a distancia, estar atentos a los cambios de comportamiento, construir nuevas rutinas ajustadas a las nuevas situaciones y procurar cuidar a los cuidadores son algunos de los puntos clave que surgen en esta situación de emergencia (Hume et al., 2020; Amaral y De Vries, 2020).

Resulta novedosa la categoría de "aislamiento social”, “confinamiento”, “cuarentena” o la denominación que se utilice en cada región. Porque nos toma por sorpresa $\mathrm{y}$, al mismo tiempo que nos exige acciones concretas como actores sociales en la comunidad, nos brinda la oportunidad de reflexionar sobre nuestras propias prácticas, cómo son percibidas por nosotros y por las familias y cuáles son las necesidades más acuciantes de las personas con TEA y sus cuidadores en este contexto de pandemia.

En ese sentido, si somos consistentes con la planificación y la intervención centrada en la persona y la familia, se hacen necesarios entonces nuevos modos de acompañar, orientar, asesorar, tomar decisiones o brindar apoyos educativos o tratamientos en las diversas áreas.

El objetivo de este trabajo es explorar la experiencia actual de la persona con TEA y sus familias. Cómo viven los días de encierro obligado, qué tipo de conductas se presentan, cuáles son sus necesidades específicas, qué tipo de tratamientos se han interrumpido y cuáles han seguido por distintas vías a distancia, qué ventajas y desventajas están presentando. Dicha exploración puede servirnos para repensar críticamente nuestras estrategias, recursos y programas de intervención tanto clínicos como educativos y, al mismo tiempo, promover la creación de nuevas propuestas centradas en las personas con TEA y sus familias en estos contextos reales y concretos. 


\section{Perspectiva global del autismo. La necesidad de cerrar brechas}

El trastorno del espectro del autismo (TEA) es un trastorno del neurodesarrollo que afecta la interacción y la comunicación social en diversos contextos, asi como la simbolización y la flexibilidad, evidenciado en patrones de comportamiento e intereses restringidos (Fuentes et al., 2020).

Desde la perspectiva global internacional del autismo se hace necesario contar con datos epidemiológicos y recursos para la detección temprana, la evaluación diagnóstica y el tratamiento en los diferentes países y no solo en los países centrales. Los estudios transculturales cobran especial importancia y el foco en los países más pobres o en "vías de desarrollo", a través de programas de cooperación o apoyos de organismos internacionales, será crucial en las próximas décadas. Los incipientes trabajos de investigación y la promoción de campañas de detección y atención temprana en países de África, Asia y Latinoamérica muestran el largo camino que queda por recorrer en la comunidad internacional en relación con las políticas públicas frente a la realidad de las personas con autismo y sus familias (Besio et al., 2018; Paula et al., 2020).

Por otra parte, como Durkin y colaboradores (2015) han expresado, la inequidad global en la investigación y conocimiento en autismo es una preocupación en sí misma. El 86 \% de la investigación acerca de TEA se hace en los países desarrollados donde solo habita el $20 \%$ de la población mundial. Además de reflejar el nivel de investigación de los países en vías de desarrollo, estas cifras son un llamado de atención sobre la información que tenemos acerca del autismo. Esta proviene de países que no comparten ni la cultura ni la formación académica de los profesionales, y mucho menos la disponibilidad de servicios. Por lo tanto, nos enfrentamos a una realidad en la cual se hace extrapolación de hallazgos que en muchos casos no son aplicables a la situación del TEA en América Latina (Grinker et al., 2011).

De acuerdo con la Organización Mundial de la Salud (Who, 2017), los especialistas en salud mental son escasos en Latinoamérica y el Caribe; lo cual tiene un impacto directo en el acceso al diagnóstico y, por lo tanto, en la derivación temprana a servicios. La carencia de profesionales capacitados en América Latina y el Caribe puede ser considerada como uno de los factores principales de la demora en el diagnóstico de TEA. Esta formación es indispensable para responder a las preocupaciones de los padres, agilizar la identificación de los TEA y su consecuente derivación temprana a servicios clínicos y educativos. Este panorama que describimos se hace aún más evidente en situaciones de emergencia como las que provoca esta pandemia.

En Latinoamérica hay aproximadamente seis millones de personas con TEA (Paula et al., 2020). Sin embargo, no hay suficiente investigación disponible en el área. En 2015, investigadores de Argentina, Brasil, Chile, República Dominicana, Uruguay y Venezuela, con el soporte técnico de Autism Speaks, formamos la Red Espectro Autista Latinoamérica (REAL) con el objetivo de realizar colaboraciones internacionales en investigaciones relacionadas con los TEA. Nos hemos centrado en la investigación de las necesidades de las personas con autismo y sus cuidadores, sus desafíos y oportunidades, las coberturas de salud, de educación, acceso a los tratamientos, organizaciones y redes de familias de personas con autismo e incidencia en políticas 
públicas. Del mismo modo, hemos estudiado dimensiones de la calidad de vida, acceso a diferentes servicios y estigmatización a causa del diagnóstico (Montiel et al., 2020). Hemos encontrado que la edad promedio de indicadores de riesgo de autismo en nuestros países es de 23 meses mientras que el diagnóstico se recibe hacia los 53 meses (Montiel et al., 2019).

Los países participantes en este estudio han comenzado con el aislamiento social, por disposición de sus gobiernos, entre mediados y fines del mes de marzo de 2020, teniendo el confinamiento diferentes características que se abordarán más adelante.

Para fines del mes de agosto de 2020, la situación de la pandemia en dichos países, en cuanto a cantidad de personas contagiadas, cantidad de fallecidos y número de personas con COVID-19 por cada 100.000 habitantes por país se muestra en la siguiente tabla.

\begin{tabular}{|l|c|c|c|}
\hline \multicolumn{3}{|c|}{ TABLA 1. Situación del COVID-19 en Latinoamérica } \\
\hline Países & Infectados & Fallecidos & $\begin{array}{c}\text { Infectados/100000 } \\
\text { habitantes }\end{array}$ \\
\hline Argentina & 736.609 & 16.591 & 742 \\
\hline Brasil & 3.605 .783 & 114.744 & 1.715 \\
\hline Chile & 488.190 & 13.529 & 2.602 \\
\hline México & 549.734 & 59.610 & 435 \\
\hline Perú & 585.236 & 27.453 & 1.793 \\
\hline Rep. Dominicana & 91.161 & 1.567 & 872 \\
\hline Uruguay & 1.543 & 43 & 223 \\
\hline Venezuela & 38.957 & 323 & 48 \\
\hline
\end{tabular}

En este contexto particular, nuestro objetivo general es describir el impacto de la pandemia -y su consiguiente confinamiento o aislamiento social- en las personas con TEA y sus familias en Latinoamérica.

Los objetivos específicos de este estudio son:

- Describir el impacto de la pandemia en personas con TEA de distintos países de Latinoamérica en relación con la conducta, el estado de ánimo, la alimentación y el sueño.

- Describir el impacto de la pandemia en los servicios, atendiendo a los distintos tipos de tratamientos y escolarización, su posible discontinuidad, la intervención telemática, sus alcances y limitaciones.

\section{Método}

Los datos fueron obtenidos de una encuesta en línea desarrollada por Red Espectro Autista Latinoamérica. Este estudio multicéntrico se realizó en 8 países: Argentina, Brasil, Chile, México, Perú, República Dominicana, Uruguay y Venezuela. 


\subsection{Muestra}

Se obtuvo una muestra intencional de cada uno de los 8 países. Se anunció a través de las redes sociales, asociaciones de padres y de profesionales, así como en instituciones que ofrecen servicios para personas con TEA. Un total de 1.852 cuidadores completaron la encuesta. De la totalidad de encuestas completadas 2 casos fueron entradas “vacías” (el sistema las guardó como encuestas completas, pero no tenían más información que el país), 4 vivían en países fuera de América Latina (España, Holanda y Nueva Zelanda). Dichos casos fueron eliminados de la base de datos antes de los análisis de resultados. El número total de casos eliminados constituyó el 0,32 \% de la muestra inicial $(n=6)$, dejando una muestra final constituida por 1.826 cuidadores. Esta muestra estuvo compuesta por un $37,1 \%(n=677)$ de Argentina, un 8,5 \% $(\mathrm{n}=156)$ de Brasil, un 13,7 \% $(\mathrm{n}=251)$ de Chile, un 6,9\% ( $\mathrm{n}=126)$ de México, un 1,8 \% (n=32) de Perú, un 9,4\% $(\mathrm{n}=171)$ de República Dominicana, un 14,2 \% $(n=259)$ de Uruguay, un 5,6 \% $(n=103)$ de Venezuela y un 2,8\% ( $n=51)$ de otros países (Bolivia, Colombia, Costa Rica, Cuba, Ecuador, Guatemala y Nicaragua). El formato de la encuesta requería ser completada en línea, y para eso los cuidadores necesitaban tener un nivel de lectura de $5 .^{\circ}$ grado, acceso a un ordenador, teléfono inteligente o dispositivo móvil e internet. En términos de sexo, un $81,8 \%(n=1493)$ eran varones, en una proporción femenino/ masculino de 1:4.5

La distribución de edades de las personas con TEA fue la siguiente: el 35,5 \% ( $\mathrm{n}=$ 726) eran menores de 6 años, el 38,9 \% (n= 710) tenían entre 7 y 12 años, el 16,8 \% entre 13 y 18 años y el 4,5\% $(n=83)$ entre 19 y 24 años. El 4,2 \% (n= 78) restante tenía edades comprendidas entre 25 y 65 años.

\begin{tabular}{|l|c|c|}
\hline \multicolumn{3}{|c|}{ TABLA 2. Distribución de participantes por país } \\
\hline \multicolumn{1}{|c|}{ Países } & $n$ & $\%$ \\
\hline Argentina & 677 & 37,1 \\
\hline Brasil & 156 & 8,5 \\
\hline Chile & 251 & 13,7 \\
\hline México & 126 & 6,9 \\
\hline Perú & 32 & 1,8 \\
\hline Rep. Dominicana & 171 & 9,4 \\
\hline Uruguay & 259 & 14,2 \\
\hline Venezuela & 103 & 5,6 \\
\hline Otros & 51 & 2,8 \\
\hline Total & 1.826 & 100 \\
\hline
\end{tabular}

Ediciones Universidad de Salamanca / CC BY-NC-ND

Siglo Cero, vol. 52, número extraordinario, 2021, pp. 119-140 


\subsection{Instrumento}

Se creó una encuesta para este estudio con la finalidad de explorar el impacto de la pandemia $-\mathrm{y}$ su consiguiente confinamiento o aislamiento social- en las personas con TEA en Latinoamérica. La encuesta está compuesta de 9 secciones:

1. Lugar de residencia y persona que completa la encuesta. De un menú en cascada los cuidadores debían escoger su país de residencia.

2. Datos generales de la persona con TEA. Esta sección incluyó: sexo, edad y diagnóstico. Para el diagnóstico se utilizaron las nomenclaturas del DSM-IV y el DSM-5 (APA, 2000, 2013).

3. Escolaridad antes y durante la pandemia, tomando en consideración modalidad de centro, nivel educativo, actividad escolar durante la pandemia, actitud de la persona con TEA frente a la actividad educativa.

4. Situación en el hogar durante la pandemia. En esta sección se incluyó información acerca del número de personas que habitaron la casa durante la pandemia, número de niños menores de 13 años residiendo en la casa. Se añadió una pregunta acerca del tamaño en metros cuadrados de la vivienda y si esta contaba con espacios exteriores para salir. Estudios recientes muestran que las características de la vivienda en términos de metros/pies o número de habitaciones y baños se correlaciona con los indicadores de salud (Dunn y Hayes, 2000). Adicionalmente, se hizo una pregunta acerca de la utilización de pantallas durante este periodo.

5. Tratamientos y apoyos al momento de iniciar la pandemia. Para esto se ofreció una lista con 16 opciones diferentes de tratamientos y apoyos. Los cuidadores podían seleccionar tantos como fueran necesarios. Luego se les preguntó si los tratamientos fueron suspendidos o modificados por la pandemia (por ejemplo, asesorías telefónicas o videollamadas), y en caso de tener tratamiento farmacológico, si había cambiado durante la pandemia.

6. Cambios conductuales a partir del aislamiento en el hogar, se consultó específicamente sobre la alimentación, sueño, agresividad, concentración, regresión de la conducta, irritabilidad, cambios en el estado de ánimo y comprensión de la prohibición de salida.

7. Salidas de la casa. Este apartado indagó acerca de las medidas de restricciones sociales en la localidad, algunas salidas o paseos implementados y sus efectos en la conducta de la persona con TEA.

8. Tratamientos y apoyos durante la pandemia. Esta sección preguntó acerca de apoyos empleados durante el confinamiento, terapias y asesoramientos a distancia, qué tratamientos se suspendieron y razones de la suspensión. Adicionalmente se solicitó a los padres indicar las ventajas y desventajas de los tratamientos a distancia, en una pregunta de respuesta abierta.

9. Principales preocupaciones durante la cuarentena, lo cual incluyó una lista de posibles preocupaciones de los cuidadores con respecto a la persona con TEA durante la pandemia. 
La encuesta se construyó en español y para su administración en Brasil fue traducida al portugués por los miembros brasileños de REAL. Una vez construida la encuesta se sometió a validación por jueces que fueron 7 profesionales (psiquiatras y psicólogos) de los diferentes países participantes. El objetivo de esta validación fue garantizar que la redacción y el uso de palabras eran apropiados para la población objeto de estudio (cuidadores). Adicionalmente, se buscó consenso para llegar a un español universal que pudiera ser usado en los diversos países de Latinoamérica. Esta encuesta fue piloteada en 3 familias de cada país para tener una idea del proceso de administración, tiempo necesario y uso de términos. En esta fase, para algunas preguntas se añadió la opción de “otro", ya que el análisis de las respuestas de los cuidadores indicó la necesidad de poder añadir información no incluida en las opciones cerradas de la encuesta.

Una vez establecida la versión final de la encuesta se subió a un servidor para ofrecer acceso en línea. Por cada país, el coordinador nacional se encargó de la implementación de la encuesta y de obtener aprobación del comité de ética. La secretaría de REAL ofreció soporte técnico para el manejo de la base de datos y solo los coordinadores nacionales tuvieron acceso a ella.

Al ingresar al portal, se les solicitaba a los cuidadores que completaran el consentimiento informado en el cual debían escoger la opción “sí” para indicar que habían leído el consentimiento informado y estaban de acuerdo en participar en la encuesta. La encuesta fue anónima, sin solicitar información personal (i. e. nombre, número de teléfono, dirección de correo). El portal transfería directamente la información del formulario a una hoja de cálculo de Excel a la que podía acceder solamente el coordinador de cada país participante.

\subsection{Análisis de los datos}

Una vez finalizada la recolección de datos (agosto, 2020), los mismos fueron codificados y unificados en una base de datos única para todos los países. La información original se recolectó usando una hoja de Excel que posteriormente fue exportada al Paquete Estadístico para las Ciencias Sociales (SPSS), Versión 26, para su análisis. El análisis de datos incluyó cálculos de tendencia central y frecuencias. Todas las variables fueron categorizadas y consideradas variables nominales.

\section{Resultados}

Tal como señalamos, uno de los objetivos que nos alentó a realizar este estudio es el de describir el impacto de la pandemia -y su consiguiente confinamiento o aislamiento social- en las personas con TEA y sus familias en Latinoamérica. Más específicamente, describir el impacto de la pandemia en personas con TEA de distintos países de Latinoamérica en relación con la alimentación, el sueño, los estados de ánimo y la conducta. Asimismo, nos hemos propuesto analizar el impacto de la pandemia en los servicios, atendiendo a los distintos tipos de terapias y escolarización, su posible discontinuidad, la intervención telemática, sus alcances y limitaciones. 
Describiremos los resultados obtenidos agrupándolos en tres categorías:

a. características sociodemográficas y habitacionales,

b. caracterización del aislamiento y restricciones sociales,

c. conductas de las personas con TEA durante el aislamiento, atendiendo a la continuidad de los servicios recibidos, tanto tratamientos como escolarización, sus estados de ánimo, cambios conductuales, alimentación y sueño.

En relación con los aspectos demográficos y habitacionales, menos del $27 \%$ de las familias de la muestra viven en casas con superficies de menos de $50 \mathrm{~m}^{2}$, mientras que el $23,5 \%$ tienen viviendas de entre 50 y $70 \mathrm{~m}^{2}$, un $26,2 \%$ viviendas de entre 70 y 100 $\mathrm{m}^{2}$ y un 23,8 \% viviendas de más de $100 \mathrm{~m}^{2}$. Casi un $80 \%$ de las familias cuenta con espacio exterior en su vivienda.

La mayoría de las familias (70 \%) encuestadas tienen uno o dos hijos menores de 13 años. El 81,5\% de las familias están compuestas por entre 3 y 5 miembros, mientras que un $11,2 \%$ están formadas por más de 5 miembros.

Un $81,8 \%$ de las personas con TEA eran varones, en una proporción femenino/ masculino de 1:4.5

La distribución de edades de las personas con TEA fue la siguiente: el 35,5 \% (n = 726) son menores de 6 años, el 38,9\% $(n=710)$ tienen entre 7 y 12 años, el $16,8 \%$ entre 13 y 18 años y el 4,5\% $(n=83)$ entre 19 y 24 años. El $4,2 \%(n=78)$ restante tienen edades comprendidas entre 25 y 65 años.

\begin{tabular}{|c|c|}
\hline \multicolumn{2}{|c|}{ TABLA 3. Datos habitacionales } \\
\hline DATOS HABITACIONALES \\
\hline Vivienda con espacio exterior & $\mathrm{n}$ \\
\hline Sí & 1449 \\
\hline No & 377 \\
\hline Cantidad de personas que viven en la casa \\
\hline 2 & 137 \\
\hline $3 / 5$ & 1485 \\
\hline$>5$ & 204 \\
\hline Familias con niños $<13$ años & 323 \\
\hline Ninguna & 716 \\
\hline 1 & 568 \\
\hline 2 & 141 \\
\hline 3 & 33 \\
\hline 4 & 45 \\
\hline$>5$ &
\end{tabular}

Ediciones Universidad de Salamanca / CC BY-NC-ND

Siglo Cero, vol. 52, número extraordinario, 2021, pp. 119-140 
En relación con el tipo de restricciones sociales o aislamiento, el 35,5 \% han tenido cuarentena obligatoria mientras que el $32,3 \%$ tuvieron cuarentena voluntaria y el $24 \%$ cuarentena obligatoria con excepciones de salidas, según la legislación y disposiciones de cada país. Casi un $48 \%$ del total de las familias respetaron el aislamiento. Algunos de sus miembr os salieron a trabajar durante el aislamiento en alrededor del $50 \%$ de los casos. La convivencia en el hogar no ha cambiado la relación entre sus miembros en un $36 \%$ de las familias, el 32,2 \% manifiestan que la relación empeoró con la convivencia mientras que para el 31,7 \% la relación ha mejorado.

\begin{tabular}{|l|c|c|}
\hline \multicolumn{2}{|c|}{ TABLA 4. Restricciones sociales } & \\
\hline Restricciones sociales & $\mathrm{n}$ & $\%$ \\
\hline Cuarentena obligatoria & 645 & $35,5 \%$ \\
\hline Cuarentena voluntaria & 586 & $32,3 \%$ \\
\hline Cuarentena intermitente & 137 & $7,5 \%$ \\
\hline Cuarentena obligatoria con excepciones & 435 & $24,0 \%$ \\
\hline Otro & 12 & $0,7 \%$ \\
\hline Salidas durante aislamiento & & \\
\hline Ningún miembro ha salido & 33 & $1,8 \%$ \\
\hline Todos permanecen en casa & 836 & $45,8 \%$ \\
\hline Uno salió a trabajar & 548 & $30,0 \%$ \\
\hline Varios miembros salen a trabajar & 160 & $8,8 \%$ \\
\hline Distintos miembros salen alternados & 232 & $12,7 \%$ \\
\hline Otro & 17 & $0,9 \%$ \\
\hline Convivencia familiar & & \\
\hline Mucho peor que antes & 95 & $5,3 \%$ \\
\hline Algo peor que antes & 481 & $26,9 \%$ \\
\hline Sin cambios & 643 & $36 \%$ \\
\hline Un poco mejor que antes & 435 & $24,3 \%$ \\
\hline Mucho mejor que antes & 133 & $7,4 \%$ \\
\hline
\end{tabular}

Con relación a la escolarización, a los tratamientos y a las conductas observadas por las familias, al momento de iniciarse la pandemia el 26,9\% de las personas con TEA recibían terapia del lenguaje, el 19,6 \% recibían medicación, el 19,5 \% terapia ocupacional, el 17,6 \% apoyo escolar, el 15,5\% terapia conductual, entre otros tipos de tratamientos. 
Casi un $30 \%$ de los tratamientos presenciales han sido suspendidos totalmente durante la pandemia. La mayor parte de las suspensiones de tratamiento han sido por cierre de centros $(28,6 \%)$ o por decisión del prestador de salud $(23,4 \%)$, mientras que en el 21,5\% de los casos no se han suspendido tratamientos. Un $40 \%$ de las familias han recibido asesoramiento a través de videollamada.

Ante la consulta sobre la preocupación de la persona con TEA por enfermarse durante la pandemia, casi un $60 \%$ no reportan cambios, mientras que un $29 \%$ muestran más preocupación. Un 40,8 \% de las personas con TEA muestran mayor nivel de dificultad para dormir. Cuando se consulta acerca de las dificultades para concentrarse, un $49,4 \%$ reportan mayor nivel de dificultad.

Un 30,2 \% manifiestan que ha habido un poco más de dificultad con la alimentación y un 15,2 \% mucha más dificultad, mientras que el 43,4 \% no registran cambios. Las familias manifiestan que el nivel de irritabilidad se incrementó en un $64,9 \%$ de los casos. El nivel de agresividad de sus hijos que registran las familias ha aumentado en el $35,5 \%$ de los casos.

Casi el $47 \%$ de las familias dicen que su hijo ha deambulado más durante la pandemia. Un 63 \% de las familias comentan que registran mayores niveles de ansiedad en su hijo durante este periodo. Según las familias, la persona con TEA ha mostrado retrocesos durante la pandemia en un 57,8 \% de los casos. Casi un $34 \%$ manifiestan que notaron avances. La mayor parte de las personas con TEA que tomaban medicación mantuvo el plan de dosis durante la pandemia. Casi la mitad de las personas con TEA mejoraron sus conductas cuando comenzaron a realizar salidas y paseos.

En relación con la escolaridad, las familias informan que un 91,1 \% tienen hijos en la escolaridad formal, de los cuales un 72,8 \% están en escuela primaria. Para la mayoría de las familias ( $75 \%$ ) de los países que componen REAL, la escolaridad se realizó a distancia durante la pandemia, mediante plataforma interactiva, a distancia con libros digitales o libros en papel. Solamente el 15,5 \% de los niños y jóvenes en edad escolar estuvieron sin actividad.

Para el $80 \%$ de las familias aumentó el uso de pantallas por parte de sus hijos.

\begin{tabular}{|l|c|c|}
\hline \multicolumn{3}{|c|}{ TABLA 5. Tratamientos } \\
\hline Tratamientos previos al inicio de la pandemia & $\mathrm{n}$ & $\%$ \\
\hline Terapia conductual & 283 & $15,5 \%$ \\
\hline Integración sensorial & 112 & $6,1 \%$ \\
\hline Rehabilitación cognitiva & 32 & $1,8 \%$ \\
\hline Terapia ocupacional & 355 & $19,5 \%$ \\
\hline Terapia física & 276 & $15,1 \%$ \\
\hline Habilidades sociales & 174 & $9,5 \%$ \\
\hline Terapia del lenguaje & 491 & $26,9 \%$ \\
\hline
\end{tabular}




\begin{tabular}{|l|c|c|}
\hline \multicolumn{3}{|c|}{ TABla 5. Tratamientos (cont.) } \\
\hline Tratamientos previos al inicio de la pandemia & $\mathrm{n}$ & $\%$ \\
\hline Medicación & 357 & $19,6 \%$ \\
\hline Terapia relacional & 36 & $2,0 \%$ \\
\hline Apoyo escolar & 320 & $17,6 \%$ \\
\hline Pedagogía & 76 & $4,2 \%$ \\
\hline Biomédico & 24 & $1,3 \%$ \\
\hline Otro & 166 & $9,1 \%$ \\
\hline Acompañamiento terapéutico & 39 & $2,1 \%$ \\
\hline Motivos de suspensión & \multicolumn{2}{|c|}{} \\
\hline Decisión del prestador & 410 & $23,4 \%$ \\
\hline Decisión de la institución & 19 & $1,1 \%$ \\
\hline No sé cuál de los dos & 58 & $3,3 \%$ \\
\hline Familia-cuidado & 246 & $14,0 \%$ \\
\hline Familia-económico & 96 & $5,5 \%$ \\
\hline Cierre del centro & 502 & $28,6 \%$ \\
\hline No se suspendió el tratamiento & 377 & $21,5 \%$ \\
\hline No tenía tratamiento & 46 & $2,6 \%$ \\
\hline Muestra avances/retrocesos & 15 & $0,8 \%$ \\
\hline Importante retroceso & 84 & $4,7 \%$ \\
\hline Algún retroceso & 538 & $30,2 \%$ \\
\hline Sin cambio & 850 & $47,7 \%$ \\
\hline Algún avance & 26 & $1,5 \%$ \\
\hline Mucho avance & 268 & $15 \%$ \\
\hline Con salidas y paseos & 164 & $31,9 \%$ \\
\hline Empeoraron la mayoría de las conductas & $25,9 \%$ \\
\hline Empeoraron algunas conductas & $9,2 \%$ \\
\hline Sin cambio & \multicolumn{2}{|c|}{} \\
\hline Mejoraron algunas conductas & $7,8 \%$ \\
\hline Mejoraron mayoría de conductas & \\
\hline No ha salido aún & \multicolumn{2}{|c|}{} \\
\hline
\end{tabular}




\begin{tabular}{|l|c|c|}
\hline \multicolumn{3}{|c|}{ TABLA 5. Tratamientos (cont.) } \\
\hline Tratamientos previos al inicio de la pandemia & $\mathrm{n}$ & $\%$ \\
\hline Escolaridad durante la pandemia & \\
\hline Sin actividad & 283 & $15,5 \%$ \\
\hline Presencial & 35 & $1,9 \%$ \\
\hline A distancia libros papel & 398 & $21,8 \%$ \\
\hline A distancia libros digital & 465 & $25,5 \%$ \\
\hline Distancia plataforma interactiva & 512 & $28,0 \%$ \\
\hline Otro & 133 & $7,3 \%$ \\
\hline Utilización de pantallas & \multicolumn{2}{|l|}{} \\
\hline Aumentó mucho & 945 & $52,2 \%$ \\
\hline Aumentó un poco & 510 & $28,2 \%$ \\
\hline Disminuyó mucho & 25 & $1,4 \%$ \\
\hline Disminuyó un poco & 44 & $2,4 \%$ \\
\hline No cambió & 286 & $15,8 \%$ \\
\hline
\end{tabular}

\begin{tabular}{|l|c|c|c|c|c|}
\hline \multicolumn{7}{|c|}{ TABLA 6. Conductas y estado de ánimo } \\
\hline & $\begin{array}{c}\text { Mucho menos que } \\
\text { antes }\end{array}$ & $\begin{array}{c}\text { Algo } \\
\text { menos }\end{array}$ & $\begin{array}{c}\text { Sin } \\
\text { cambio }\end{array}$ & $\begin{array}{c}\text { Un poco } \\
\text { más }\end{array}$ & $\begin{array}{c}\text { Mucho } \\
\text { más }\end{array}$ \\
\hline $\begin{array}{l}\text { Preocupación } \\
\text { por enfermarse }\end{array}$ & $\begin{array}{c}111 \\
6,1 \%\end{array}$ & $\begin{array}{c}112 \\
6,1 \%\end{array}$ & $\begin{array}{c}1077 \\
59 \%\end{array}$ & $\begin{array}{c}396 \\
21,7 \%\end{array}$ & $\begin{array}{c}130 \\
7,1 \%\end{array}$ \\
\hline Dificultad \\
para dormir & 59 & 60 & 961 & 521 & 225 \\
\hline Concentrarse & $3,2 \%$ & $3,3 \%$ & $52,6 \%$ & $28,5 \%$ & $12,3 \%$ \\
\hline Alimentación & 89 & 152 & 674 & 638 & 257 \\
& $4,9 \%$ & $8,4 \%$ & $37,2 \%$ & $35,2 \%$ & $14,2 \%$ \\
\hline Irritabilidad & $6,4 \%$ & $7,9 \%$ & $43,4 \%$ & $30,2 \%$ & $15,2 \%$ \\
\hline Agresión & 84 & 116 & 425 & 796 & 363 \\
& $4,7 \%$ & $6,5 \%$ & $23,8 \%$ & $44,6 \%$ & $20,3 \%$ \\
\hline Deambular & 119 & 53 & 990 & 456 & 184 \\
& $7,6 \%$ & $2,9 \%$ & $54,9 \%$ & $25,3 \%$ & $10,2 \%$ \\
\hline Alteraciones del & $4,0 \%$ & $2,9 \%$ & $46,4 \%$ & $29,8 \%$ & $16,8 \%$ \\
\hline estado de ánimo & 52 & 66 & 846 & 605 & 215 \\
\hline Ansiedad & $2,9 \%$ & $3,7 \%$ & $47,4, \%$ & $33,9 \%$ & $12,1 \%$ \\
\hline & 42 & 57 & 632 & 757 & 301 \\
& $2,3 \%$ & $3,2 \%$ & $35,3 \%$ & $42,3 \%$ & $16,8 \%$ \\
\hline
\end{tabular}




\section{Discusión}

La prevalencia de casos de TEA ha aumentado significativamente en el mundo, incluyendo los países de Latinoamérica y el Caribe (Elsabbag et al., 2015). Del mismo modo aumentan los casos de COVID-19 de manera global. Por ello se hace necesario investigar acerca del impacto de la pandemia y su consecuente aislamiento social (en diversos formatos según disposiciones de cada país) sobre las personas con TEA y sus familias. Por otra parte, ante la incertidumbre futura sobre el alcance y duración de la pandemia y las medidas sanitarias a adoptar, consideramos importante valorar los datos obtenidos para ser capaces de crear nuevas propuestas vinculadas con los dispositivos de apoyo en los diferentes servicios de salud y educación de los países incluidos en este estudio.

Es sabido que la salud mental, la calidad de vida y el bienestar emocional se han visto afectados en toda la población de manera global. Pero las personas con discapacidad y sus familias presentan mayor nivel de vulnerabilidad ante este tipo de crisis sanitarias (Holmes et al., 2020). En un estudio reciente en Reino Unido, tanto los padres como sus propios hijos con discapacidad experimentan sentimientos de pérdida, de preocupación y cambios en el estado de ánimo y la conducta frente al impacto del COVID-19 (Asbury et al., 2020). Por otra parte, cabe destacar los altos niveles de coocurrencia de otras condiciones de salud física y mental en las personas con autismo (problemas gastrointestinales y de sueño, ansiedad, depresión, por ejemplo). Estos datos van en la misma línea que los resultados que hemos obtenido en este estudio. Un análisis preliminar de datos de registros médicos globales (hasta el 14 de mayo de 2020) muestra que personas con discapacidad intelectual y del desarrollo tienen mayor prevalencia de comorbilidades físicas específicas asociadas con un peor pronóstico para el COVID-19. Además, entre las personas jóvenes (menores de 17 años), aquellos con discapacidad intelectual tienen mayor probabilidad de ser infectados y experimentar porcentajes más elevados de mortalidad que aquellos sin discapacidad intelectual (Ameis et al., 2020). Un fenómeno particular que se ha evidenciado con el confinamiento en los hogares es que se tornan invisibles ciertas injusticias e inequidades (Blow, 2020). Esto se destaca en los países estudiados, en los que las decisiones políticas han prescripto el confinamiento de las familias en sus hogares. Pellicano y Stears puntualizan (2020) que las profundas dificultades que enfrentan muchas personas con autismo no atraen la atención del público, y que las organizaciones de familias que defienden sus derechos, los grupos comunitarios y los investigadores encuentran más obstáculos en tiempos de pandemia para sacar a la superficie temas cruciales como lo han hecho en otras oportunidades. Algunas de las situaciones extremas ocultas por la pandemia se vinculan con experiencias de abuso y exclusión de los cuidados y servicios adecuados, que se estima que se han incrementado comparadas con el riesgo reportado en situaciones comunes. Tal es el caso de la suspensión de los tratamientos en Latinoamérica y la consecuente prohibición a los terapeutas y personal de apoyo escolar de realizar actividad presencial con las personas con TEA (Weiss y Fardella, 2018). 
Es sabido además que las desigualdades económicas se han profundizado con las medidas tomadas frente al COVID-19, que han incrementado los problemas laborales y la pobreza. Las consecuencias futuras de la presente crisis resultan aún desconocidas, aunque se estiman graves y duraderas. El acceso y el uso de servicios y la presencia de la estigmatización en distintas regiones geográficas se ven influidos por las diferencias socioculturales y los niveles socioeconómicos, por ejemplo, países latinoamericanos con poblaciones de ingresos medios y bajos (Durkin et al., 2015; Magana et al., 2013; Mandell et al., 2009). Asimismo, factores sistémicos como la falta de recursos en áreas rurales, falta de coberturas de salud y niveles educativos de las familias influyen en el tipo y variedad de tratamiento al que suelen tener acceso (Lindly et al., 2019).

La profundidad de la brecha tecnológica también se ha puesto de manifiesto frente a la necesidad de continuar con tratamientos y escolaridad a través de pantallas vía internet. Muchos hogares de Latinoamérica no están preparados para la magnitud de transferencia de datos necesarios o bien no poseen cobertura para conexiones de internet. Del mismo modo, se sabe que hay familias con varios niños en edad escolar y no cuentan con tantos dispositivos para conectarse y continuar con sus tareas, incluido el teletrabajo de algunos padres y madres, tal como reportan en nuestra encuesta al ser consultados sobre sus trabajos.

Para comprender los desafíos que enfrentan las familias de personas con TEA en Latinoamérica es crucial entender su contexto social, económico y cultural. Para este estudio hemos presentado datos de Argentina, Brasil, Chile, México, Perú, República Dominicana, Uruguay y Venezuela. Todos estos países comparten herencia cultural con España o Portugal mezclada con culturas de comunidades aborígenes nativas de América o África (Padilla et al., 2020). La inequidad en los sistemas de salud es común para las comunidades en situación de pobreza y las poblaciones más vulnerables por ejemplo, mujeres, niños, adolescentes y población aborigen (UNICEF, 2016; World Bank, 2015). Vale la pena destacar que, según las estadísticas internacionales, los países participantes de la muestra cuentan con un número de profesionales de la salud mental por debajo del promedio estándar por cantidad de habitantes (Who, 2017). Algunos de estos países que se suponen con altos ingresos son en realidad países de ingresos medios y bajos y gran parte de la población se enfrenta a situaciones de pobreza, inequidad e inseguridad. Por ejemplo, en la década pasada, Venezuela ha padecido el mayor nivel de inflación del mundo (2297 \%), mientras Argentina ocupó el quinto lugar $(42,1 \%)$ y Uruguay el vigésimo $(11,05 \%)$ (International Monetary Fund, 2020). Para disminuir la inequidad en salud en países con dichas circunstancias políticas y económicas y con una creciente prevalencia de TEA, datos confiables, evidencia sólida y conocimiento riguroso deben remplazar al oscurantismo, la ideología regresiva y la ineficacia en el proceso de toma de decisiones (UNICEF, 2016). Este enfoque es aplicable a todos los aspectos relacionados con la salud, incluyendo el TEA y el COVID-19.

Una de las primeras cuestiones que surgen de un análisis global de los datos es que en algunas áreas casi la mitad de las personas con TEA no registran importantes cambios, mientras que en otros aspectos el impacto del encierro es más notable. 
¿Cómo se explica que algunas personas con autismo hayan disfrutado de la situación de confinamiento mientras otras hayan empeorado su conducta o incluso mostrado señales de retroceso según su familia? Puede deberse en parte a la propia heterogeneidad que presenta el cuadro en cada persona. Hay otros factores que pueden incidir en las formas de afrontar la experiencia de confinamiento. Mientras que para algunas personas con autismo la exposición a los estímulos del mundo exterior al hogar puede constituir fuente de estrés, otras personas necesitan de paseos y de cambios de ambiente, sobre todo cuando la situación de encierro no ofrece estructuras de actividades que contribuyan a la regulación de la conducta (Nalbant, 2018; Toscano et al., 2018).

La escuela es fuente de oportunidades de aprendizaje e interacción con otras personas. También es un desafío para la autorregulación, la organización del tiempo y el espacio y el manejo de la conducta. Para algunos estudiantes no asistir a la escuela podría provocar niveles más altos de ansiedad y desorganización, mientras que para otros quedarse en casa puede significar estar menos expuesto a situaciones nuevas y cambiantes y demás exigencias cognitivas y sociales que promueve el contexto escolar, tal como comentan algunas de las familias.

En Estados Unidos una reciente encuesta a 818 familias con hijos con discapacidad intelectual o del desarrollo encuentra que el $74 \%$ de los padres reportan que su hijo ha perdido el acceso al menos a un servicio terapéutico o educativo (Jeste et al., 2020).

Otro claro reto lo constituye la modalidad de intervención remota, ya que, si bien algunas personas con autismo se han beneficiado de ese recurso para continuar sus terapias o su escolaridad, para otras personas con autismo no verbales o mínimamente verbales, el uso de pantallas puede suponer desafíos en sus niveles de atención, concentración, motivación. Incluso en personas con autismo, lenguaje verbal e inteligencia promedio, sin mayores dificultades para apropiarse de contenidos del currículum formal, la demanda social y las variables relativas al currículum oculto. Este último incluye valores y conductas implícitas, convenciones y normas específicas del dispositivo escolar (Valdez et al., 2016; Valdez, 2020) que constituyen barreras, en ocasiones muy difíciles de franquear, tal como plantea Reicher (2020) al analizar el aprendizaje remoto durante la pandemia en niños con TEA de "alto funcionamiento". Algunos datos complementarios en primera persona, aportados por las propias personas con TEA, forman parte de un estudio que se encuentra en preparación, subrayando la importancia de atender las propias percepciones de personas con autismo que han podido expresar sus propios puntos de vista (Núñez et al., en preparación; Valdez et al., 2020).

Datos preliminares de un estudio italiano (Colizzi et al., 2020) destacan el impacto negativo sobre las familias con hijos con autismo, incluyendo los desafíos cotidianos para gestionar actividades estructuradas y tiempo libre y un aumento de problemas de regulación de la conducta en los niños. Padres de niños con autismo en Turquía también reportaron niveles altos de ansiedad y bajos niveles de bienestar emocional comparados con padres de niños neurotípicos.

Nuestros datos muestran que algunas de las consecuencias del confinamiento han sido el aumento de irritabilidad en las personas con TEA (64,9\%), el incremento de 
la conducta de deambular (47\%), mayores niveles de ansiedad (63\%) y de conductas relacionadas con la agresividad (35\%).

Por otra parte, un $57,8 \%$ de los padres han notado retrocesos en sus hijos durante la etapa de encierro.

Es sabido también que durante el confinamiento la continuidad de los tratamientos, aunque por vía telemática, ha favorecido la organización de la vida cotidiana de la persona con autismo. Un efecto no buscado pero que resulta interesante para futuros análisis es el asesoramiento sistemático que han tenido muchas familias para mejorar el manejo de problemas de conducta en situaciones de la vida cotidiana, ante la imposibilidad de la intervención en contextos reales de los propios terapeutas y personal de apoyo.

En nuestra encuesta se han realizado preguntas abiertas sobre las ventajas y desventajas de los "teletratamientos". Mayoritariamente las familias destacaron sus desventajas, haciendo énfasis en que las dificultades de atención y concentración de sus hijos, como la hiperactividad, hicieron casi imposible la relación con docentes y terapeutas a través de pantallas. Otros subrayaron la necesidad de interacción y contacto personal que las pantallas no pueden remplazar. Del mismo modo varias familias plantearon que termina siendo una sobrecarga para los padres, que se suma a la actividad cotidiana, al trabajo y a la escolarización; agregando además que muchos de ellos no se sienten capaces de llevar adelante terapias por no ser expertos. Puntualizaron también que a muchos terapeutas les faltaba experiencia para manejar la tecnología y esto supone una barrera para este tipo de tratamientos.

Las familias que encuentran algunas ventajas en el "teletratamiento" enfatizaron que sus hijos están menos sometidos a estrés por los viajes y las exigencias de la interacción social, destacaron que de ese modo ellos pueden involucrarse más como padres, comprender mejor los tratamientos y tener una relación más cercana y personal con los terapeutas. Este tipo de reflexiones deberían ayudarnos a repensar las relaciones entre familias y profesionales en tiempos de "normalidad" y cómo los abordajes terapéuticos incluyen o no a las familias en sus objetivos, sus estrategias y sus propuestas (Reicher, 2020; Dahiya et al., 2020).

Es importante destacar que casi la mitad de las personas con TEA han mejorado sus conductas cuando comenzaron a realizar salidas y paseos.

Respecto de la eficacia de los paseos o salidas, es fundamental tener en cuenta que muchas de las personas con autismo necesitan estas rutinas de ocio de manera particular (ejercicio físico, caminatas, correr o saltar en espacios libres) (ver Esenturk, 2020; Yarimkaya y Esenturk, 2020). Para muchas personas con TEA constituye un desafío organizar el tiempo de las rutinas cotidianas. El paseo o salida requiere anticipación al cambio de actividad y de lugar físico, promueve la competencia ejecutiva de dar sentido a una actividad, junto a preparativos prácticos relativos a higiene, vestimenta, etc. Para muchas personas con autismo estos desafíos se han constituido en logros cuya pérdida puede significar un retroceso o pausa en estos aprendizajes. La falta de salidas recreativas, y el consecuente tiempo sin propósito o no dirigido a metas, puede derivar en incremento de conductas disfuncionales y malestar psicológico. La pandemia ha puesto de manifiesto la necesidad de preservar las salidas o paseos diarios para 
personas con autismo y será importante para las autoridades tener en cuenta estos datos a fin de asegurar este tipo de salidas si en el futuro el confinamiento se extiende en el tiempo.

El estudio que aquí presentamos se circunscribe a la descripción de datos globales, que pueden ser de utilidad para comprender el alcance de las intervenciones clínicas y educativas durante el aislamiento social y sus efectos en las personas con TEA y sus familias (cfr. propuestas como las de Tarbox et al., 2020; Valdez et al., 2020; Amigo et al., 2020). Sin embargo, somos conscientes de que a estos primeros datos han de seguirles otros análisis que den cuenta de las interacciones entre variables de tratamiento, escolaridad y paseos y su correlación con conductas, ansiedad, alimentación y sueño, por ejemplo, en grupos de edades diferentes y en diferentes países con formas de restricción social distintas (Aishworiya y Kang, 2020).

Muchos de estos datos ponen de relieve la importancia del enfoque ecológico del desarrollo humano (Bronfenbrenner, 1987) y las relaciones entre decisiones políticas (macrosistema) y su impacto en el desarrollo y bienestar emocional del niño y su familia (microsistema). Este planteamiento, como puede notarse, no es meramente teórico, sino que tiene importantes consecuencias prácticas y nos ayuda a comprender mejor la importancia de los apoyos en contextos funcionales y los vasos comunicantes entre el hogar, la escuela, las terapias, el barrio y la comunidad (mesosistema).

La presente crisis mundial debería servir como una oportunidad para hacernos reflexionar sobre los sistemas de salud y educación y la necesidad de cambios, con una perspectiva más ecológica, inclusiva y amigable con el autismo. Muchos de los cambios hechos sobre la marcha, en la medida que impliquen experiencias positivas, deberían ser útiles para crear estrategias futuras para afrontar a mediano y largo plazo circunstancias tan complejas como la que la pandemia de COVID-19 ha producido (Luckasson y Schalock, 2020).

En definitiva, más allá de la situación de emergencia, para la persona con autismo, bienestar emocional y calidad de vida significan, mediante los apoyos necesarios, multiplicar las posibilidades de participación en diversidad de contextos para una inclusión plena en la comunidad.

\section{Referencias bibliográficas}

Aishworiya, R. y Kang, Y. Q. (2020). Including children with developmental disabilities in the equation during this COVID-19 pandemic. Journal of Autism and Developmental Disorders, 1-9. Advance online publication. https://doi.org/10.1007/s10803-020-04670-6

Amaral, D. G. y De Vries, P. J. (2020). COVID-19 and autism research: perspectives from around the globe. Autism Research, 13(6), 844. https://doi/10.1002/aur.2329

Ameis, S., Lai, M., Mulsant, B. y Szatmari, P. (2020). Coping, fostering resilience, and driving care innovation for autistic people and their families during the COVID-19 pandemic and beyond. Molecular Autism, 11, 61. https://doi.org/10.1186/s13229-020-00365-y

American Psychiatric Association. (2000). Diagnostic and statistical manual of mental disorders (4th ed., Text Revision). 
American Psychiatric Association. (2013). Diagnostic and statistical manual of mental disorders (5th ed.). American Psychiatric Publishing.

Amigo, C., Suárez, J., Segura, M. y Garrido, G. (2020). Estrategias de apoyo para personas con TEA y sus familias durante la pandemia por COVID-19. http://www.unidadteauruguay.org/images/uploads/Estrategias\%20de\%20apoyo $\% 20$ para $\% 20$ personas $\% 20$ con $\% 20$ TEA $\% 20$ y $\% 20$ sus $\% 20$ familias, $\% 20$ durante $\% 201$ a $\% 20$ pandemia $\% 20$ por $\% 20$ COVID-19.pdf

Asbury, K., Fox, L., Deniz, E., Code, A. y Toseeb, U. (2020). How is COVID-19 affecting the mental health of children with special educational needs and disabilities and their families? Journal of Autism and Developmental Disorders, 1-9. Advance online publication. https://doi.org/10.1007/s10803-020-04577-2

Becker, S. P. y Gregory, A. M. (2020). Editorial perspective: perils and promise for child and adolescent sleep and associated psychopathology during the COVID-19 pandemic. Journal of Child Psychology and Psychiatry, 61(7), 757-759. https://doi:10.1111/jcpp.13278

Besio, V., Garrido, G., Montiel-Nava, C., García Sepúlveda, R., Valdez, D., Rosoli, A., Cukier, S., Paula, C., Irrazava, M. y Rattazzi, A. (2018). Financial impact, quality of life and stigma in Uruguayan families of patients with Autism Spectrum Disorder in the framework of a new mental health law. Póster. INSAR.

Blow, C. M. (2020). Social distancing is a privilege. The New York Times. https://www.nytimes.com/2020/04/05/opinion/coronavirus-social-distancing.html

Bronfenbrenner, U. (1987). La ecología del desarrollo bumano. Paidós.

Colizzi, M., Sironi, E., Antonini, F., Ciceri, M., Bovo, C. y Zoccante, L. (2020). Psychosocial and behavioral impact of COVID-19 in Autism Spectrum Disorder: an online parent survey. Brain Sciences, 10, 341. https://doi:0.3390/brainsci10060341

Dahiya, A. V., McDonnell, C., DeLucia, E. y Scarpa, A. (2020). A systematic review of remote telehealth assessments for early signs of autism spectrum disorder: video and mobile applications. Practice Innovations, 5(2), 150-164. https://doi.org/10.1037/pri0000121

Degli Espinosa, F., Metko, A., Raimondi, M., Impenna, M. y Scognamiglio, E. (2020). A model of support for families of children with autism living in the COVID-19 lockdown: lessons from Italy. Behavior Analysis in Practice, 13, 550-558. https://doi.org/10.1007/ s40617-020-00438-7

Dunn, J. y Hayes, M. (2000). Social inequality, population health, and housing: a study of two Vancouver neighborhoods. Social Science and Medicine, 51(4), 563-587 [PubMed: 10868671].

Elsabbagh, M., Divan, G., Koh, Y. J., Kim, Y. S., Kauchali, S., Marcin, C., Montiel-Nava, C., Patel, V., Paula, C. S., Wang, C., Yasamy, M. T. y Fombonne, E. (2012). Global prevalence of autism and other pervasive developmental disorders. Autism Research, 5(3), 160-179. https://doi.org/10.1002/aur.239

EsEntúrK, O. K. (2020). Parents' perceptions on physical activity for their children with autism spectrum disorders during the novel coronavirus outbreak. International Journal of Developmental Disabilities. https://doi:10.1080/20473869.2020.1769333

Ferreira, J., Toscano, CH., Machado-Rodrigues, A., Furtado, G., Barros, M., Souza, R. y Carvalho, H. (2018). Study protocol: effects of a physical exercise program (PEPAut) on children's with autism stereotyped behaviour metabolic and physical activity profiles, physical fitness and health related quality of life. Frontiers in Public Health, 6. https:// doi.10.3389/fpubh.2018.00047. 
Franz L., Chambers N., Von Isenburg M. y De Vries P. J. (2017). Autism spectrum disorder in Sub-Saharan Africa: a comprehensive scoping review. Autism Research, 10(5), 723 749. https://doi.10.1002/aur.1766

Fuentes, J., Hervás, A. y Howlin, P. (2020). Guía práctica para el autismo de ESCAP: Resumen de las recomendaciones basadas en la evidencia para su diagnóstico y tratamiento. (Grupo de Trabajo de ESCAP para el Autismo) - European Child and Adolescent Psychiatry. https://doi.org/10.1007/s00787-020-01587-4

Holmes, E. A., O’Connor, R. C., Hugh Perry, V., Tracey, I., Wessely, S., Arseneault, L., Ballard, C., Christensen, H., Silver, R. C., Everall, I., Ford, T., John, A., Kabir, T., King, K., Madan, I., Michie, S., Przybylski, A. K., Shafran, R., Sweeney, A.,... y Bullmore, E. (2020). Multidisciplinary research priorities for the COVID-19 pandemic: a call for action for mental health science. The Lancet Psychiatry. Advance online publication. https://doi.org/10.1016/S2215-0366(20)30168-1

InTERnATIONAL MONETARY Fund. (2020). Inflation rate, average consumer prices. Recuperado 06/15/2020 de https://www.imf.org/external/datamapper/PCPIPCH@WEO/OEMDC

Jeste, S., Hyde, C., Distefano, C., Halladay, A., Ray, S., Porath, M., Wilson, R. B. y Thurm, A. (2020). Changes in access to educational and healthcare services for individuals with intellectual and developmental disabilities during COVID-19 restrictions. Journal of Intellectual Disability Research, 64, 825-833. https://doi.org/10.1111/jir.12776

Johnson, M. D., Urm, S. H., Jung, J. A., Yun, H. D., Munitz, G. E., Tsigrelis, C., Baddour, L. M. y Juhn, Y. J. (2013). Housing data-based socioeconomic index and risk of invasive pneumococcal disease: an exploratory study. Epidemiology and infection, 141(4), 880-887. https://doi.org/10.1017/S0950268812001252

Lindly, O. J., Zuckerman, K. E. y Kuhlthau, K. A. (2019). Healthcare access and services use among US children with autism spectrum disorder. Autism, 23(6), 1419-1430. https:// doi.org/10.1177/1362361318815237

Luckasson, R. y Schalock, R. L. (2020). A balanced approach to decision-making in supporting people with IDD in extraordinarily challenging times. Research in Developmental Disabilities, 105, 103719. https://doi.org/10.1016/j.ridd.2020.103719

Magana, S., Lopez, K., Aguinaga, A. y Morton, H. (2013). Access to diagnosis and treatment services among Latino children with autism spectrum disorders. Intellectual Developmental Disability, 51(3), 141-153. https://doi.org/10.1352/1934-9556-51.3.141

Mandell, D. S., Wiggins, L. D., Carpenter, L. A., Daniels, J., Diguiseppi, C., Durkin, M. S., Giarelli, E., Morrier, M. J., Nicholas, J. S., Pinto-Martin, J. A., Shattuck, P. T., Thomas, K. C., Yeargin-Allsopp, M. y Kirby, R. S. (2009). Racial/ethnic disparities in the identification of children with autism spectrum disorders. American Journal Public Health, 99(3), 493-498. https://doi.org/10.2105/AJPH.2007.131243

Montiel-Nava, C., Cukier, S., Garrido, G., Valdez, D., Paula, C., García, R., Rosoli, A., Irarrazaval, M. y Rattazzi, A. (2020). Service encounters across the lifespan in individuals with autism spectrum disorders: results from a multisite study in Latin America. Research in Autism Spectrum Disorders. https://doi.10.1016/j.rasd.2020.101670

Nalbant, S. (2018). Effects of participation in inclusive physical activity on social skills of individuals with Autism Spectrum Disorder. Journal of Education and Training Studies, 6(12), 255-261. https://dx.doi.org/10.11114/jets.v6i12.3789

Ne'eman, A. (2020). How governments should maintain disability services during the pandemic. Spectrum News. https://www.spectrumnews.org/opinion/viewpoint/ how-governments-should-maintain-disability-services- during-the-pandemic/ 
D. VALDEZ, C. MONTIEL, C. SILVESTRE, A. RATTAZZI, A. ROSOLI, N. BARRIOS, S. CUKIER, R. GARCÍA...

Núñez, M., Valdez, D., Rattazzi, A., Cukier, S., Barrios, N. y Montiel, C. (en preparación). The impact of the lockdown due to COVID-19 on ASD people in Argentina: a first person point of view.

Padilla, J., Jager, J., Updegraff, K. A., McHale, S. M. y Umana-Taylor, A. J. (2020). Mexican-origin family members' unique and shared family perspectives of familism values and their links with parent-youth relationship quality. Developmental Psychology, 56(5), 993-1008. https://doi.org/10.1037/dev0000913

Paula, C., Cukier, S., Irarrazabal, M., Montiel-Nava, C., Garcia, R., Rosoli, A., Valdez, D., Bordini, D., Shih, A., Garrido, G. y Rattazzi, A. (2020). Challenges, priorities, barriers to care, and stigma in families of people with autism: Similarities and differences among six Latin American countries. Autism, 1-15. sagepub.com/journals-permissions. https://doi:10.1177/1362361320940073journals.sagepub.com/home/aut

Pellicano, E. y Stears, M. (2020). The Hidden Inequalities Of Covid-19. Autism2020, 24(6), 1309-1310. https://doi:10.1177/13623/32092759059

Reicher, D. (2020). Debate: remote learning during COVID-19 for children with high functioning autism spectrum disorder. Child Adolesc Ment Health, 25(4), 263-264.. https:// doi:10.1111/camh.12425

Samms-Vaughan, M., Rahbar, M. H., Dickerson, A. S., Loveland, K. A., Hessabi, M., Pearson, D. A., Bressler, J., Shakespeare-Pellington, S., Grove, M. L., Coore-Desai, C., ReEce, J. y Boerwinkle, E. (2017). The diagnosis of autism and autism spectrum disorder in low- and middle-income countries: experience from Jamaica. Autism, 21(5), 564-572. https://doi.org/10.1177/1362361317698938

Simmons, A. L. (2020). COVID-19 social distancing: a snippet view of the autistic social world. Disability and Society, 35, 6, 1007-1011. https://doi:10.1080/09687599.2020.1774866

Tarbox, C. M., Silverman, E. A., Chastain, A. N., Little, A., Bermudez, T. L. y Tarbox, J. (2020). Taking ACTion: 18 simple strategies for supporting children with autism during the COVID-19 Pandemic. Behavior Analysis in Practice, 1-29. Advance online publication. https://doi.org/10.1007/s40617-020-00448-5

Toscano, C., Carvalho, H. y Ferreira, J. (2018). Exercise effects for children with Autism Spectrum Disorder: metabolic health, autistic traits, and quality of life. Percept Mot Skills, 125(1), 126-146. https://doi:10.1177/0031512517743823

UnICEF. (2016). Health Equity Report 2916. Analysis of reproductive, maternal, newborn, child and adolescent health inequities in Latin America and the Caribbean to inform policymaking (978-92-806-4842-3).

VAldez, D. (2020). Contextos amigables con el autismo. Hilos y colores de su entramado. Autismo Ávila.

Valdez, D., Gómez, L. y Cuesta, J. L. (2016). Inclusive education and autism spectrum disorders: the working practice of support teachers in Argentina. En B. REICHOw, B. BoyD, E. E. Barton y S. L. Odom (Eds.), Handbook of Early Childhood Special Education (pp. 473-486). Springer.

Valdez, D., Manrique, S., Cañote, S., Lazo, C., Canales, Ch. y Solcoff, K. (2020). Apoyos a las personas con condiciones del espectro autista y sus familias en tiempos difíciles. Documento de libre descarga FLACSO Argentina y CPAL Perú. https://www.aprendizaje. flacso.org.ar/post/mequedoencasa-apoyo-a-las-personas-con-condiciones-del-espectroautista-y-sus-familias-en-tiempos?fbclid=IwAROytpPzicavTB89oogxMuiJF1bsDU2Cjh CPBK_9muBeEmtBiZvtGxkatPM 
Valdez, D., Martos, J., Llorente, M. y Solcoff, K. (2020). Autismo en primera persona. Los desafíos de Ángel Rivière. Miradas sobre el autismo en 2020: Monografía homenaje a Ángel Rivière. Journal for the Study of Education and Development/Infancia y Aprendizaje, 43(4).

Valdez, D., Rattazzi, A., Paula, C., García Sepúlveda, R., Garrido, G., Rosoli, A., CuKier, S., Barrios, N. y Montiel-Nava, C. (2019). Closing gaps in autism. Implementation of the Latin American Autism Spectrum network caregiver needs survey. Conference: Autism Europe 2019. https://doi:10.13140/RG.2.2.18320.23043

Weiss, J. y Fardella, M. (2018). Victimization and perpetration experiences of adults with autism. Frontiers in Psychiatry, 9, Article 203. https://doi.org/10.3389/fpsyt.2018.00203

Wilson, K. M., Klein, J. D., Blumkin, A. K., Gottlieb, M. y Winickoff, J. P. (2011). Tobacco-smoke exposure in children who live in multiunit housing. Pediatrics, 127(1), 85-92. [PubMed: 21149434].

World BAnk. (2015). The World Bank Annual Report 2015. http://hdl.handle.net/10986/22550

World BAnk. (2017). World Bank Annual Report 2017. http://documents.worldbank.org/curated/en/143021506909711004/World-Bank-Annual-Report-2017

World Health Organization. (2017). Mental Health ATLAS 2017. https://www.who.int/ mental_health/evidence/atlas/profiles-2017/en/

World Health Organization. (2018). Mental Health Atlas 2017. Country Profiles. https:// www.who.int/mental_health/evidence/atlas/profiles-2017/en/

Yarimkaya, E. y Esentúrk, O. K. (2020). Promoting physical activity for children with autism spectrum disorders during Coronavirus outbreak: benefits, strategies, and examples. International Journal of Developmental Disabilities. https://doi:10.1080/20473869.2020.1 756115 